\title{
A critical review of autism spectrum disorders in CHARGE syndrome
}

\author{
Vaivre-Douret $L^{1,2,3,4,5,6^{*}}$, Hamiaux $P^{1,2,7}$ and Abadie $V^{1,2,7}$ \\ ${ }^{1}$ Faculty of Medicine, University of Paris Descartes, Sorbonne Paris City (SPC), Paris, France \\ ${ }^{2}$ INSERM Unit 1178/1018-CESP, University of Paris Sud-Paris Saclay, UVSQVillejuif and Paris Descartes, SPC ; Necker-Enfants-Malades University Hospital, \\ AP-HP, Paris, France \\ ${ }^{3}$ Department of Child Psychiatry, Necker-Enfants Malades University Hospital, AP-HP, Paris, France \\ ${ }^{4}$ Department of Pediatrics, AP-HP Paris Center Port Royal-Cochin Hospital, Paris, France \\ ${ }^{5}$ Hospitalo University Institute Imagine, Paris, France \\ ${ }^{6}$ University Institut of France (IUF), Paris, France \\ ${ }^{7}$ Department of Paediatrics, Necker-Enfants Malades University Hospital, AP-HP, Paris, France
}

\begin{abstract}
The aim of this manuscript is to analyze the literature regarding autism spectrum disorders (ASDs) in CHARGE syndrome. Systematic search of articles in SCIENCE DIRECT, MEDLINE and PsycINFO databases. Articles published in English and French were searched with the terms "autis", "behavior", "development" associated with "CHARGE syndrome". From 247 citations, we selected 16 relevant papers, then included 7 articles in the qualitative synthesis. Data were extracted on methodological quality (use of ASD-specific tools and measurements of factors), number of participants and results.

Few studies investigated ASDs in CHARGE syndrome and even fewer used specific or diagnostic tools for ASDs. Rates are inconsistent across studies, which might be due to variation in tools used, from diagnostic tools to parental reports. Factors of ASDs in CHARGE syndrome included cerebral anomalies, degree of visual and hearing loss, intellectual disability, and medical involvement. Results are inconsistent and may be due to different ways of accounting for sensory impairments and ASDs or overlap among factors. CHARGE syndrome could exhibit a specific autistic-like profile even if few studies have precisely investigated autistic behaviors in this population. Challenging behaviors and autistic-like behaviors are often confused in patients with CHARGE syndrome. Future research is needed in both areas
\end{abstract}

\begin{abstract}
Abbreviations: ABC: Autism Behavior Checklist; ADOS: Autism Diagnostic Observation Schedule; ADI: Autism Diagnostic Interview; ASDs: Autism Spectrum Disorders; CARS: Childhood Autism Rating Scale; CS: CHARGE syndrome; DSM: Diagnostic and Statistical Manual of Mental Disorders; OAV: Oculo-Auriculo-Vertebral syndrome; PDD: Pervasive Developmental disorder; VABS: Vineland Adaptive Behavior Scale.
\end{abstract}

\section{Introduction}

CHARGE syndrome (CS) $[1,2]$ is a rare genetic syndrome $(1 / 10,000$ people) that can feature multiple disabilities. CHARGE is an acronym for the most common impairments [3]: C: coloboma (visual impairment), $\mathrm{H}$ : heart disease, A: choanal atresia, R: retarded growth and/or development, G: genital hypoplasia, E: ear anomalies or deafness. Other possible malformations and deficits include arhinencephly resulting in hyposmia, anomalies of the semicircular canals resulting in vestibular dysfunction, and cranial nerve dysfunction resulting in feeding and respiratory difficulties during the first years of life. The associations and degree of impairments vary among people with CS. Intellectual abilities are heterogeneous and could be linked to visual and neurologic anomalies [4].

Obviously, CS may lead to behavioral disorders. People with CS seem to have difficulties making same-age friendships even during the teenage years and adulthood [5-6]. They can also show ritualistic behaviors (e.g., must do things in a certain order) [7] and unusual behaviors (e.g., unusual interest in numbers, strings) [7-8], sensory particularities (e.g., desire for pressing or tight clothing) and selfstimulation behaviors [7,9]. These descriptions suggest autistic-like behaviors. The autistic quality of these behaviors has been investigated and has been reported in different studies using different methods.

Autism spectrum disorders (ASDs) are characterized by deficits in communication and social interactions as well as repetitive and restricted behaviors and interests and can vary among patients [DSM-5] [10]. Hence, ASDs include autism, Asperger's syndrome and pervasive developmental disorders [DSM-IV-TR] [11]. ASDs in CS have been described by parents [12], by clinicians using different diagnostic criteria [DSM-III] [13], DSM-IV] [11], and with various parental questionnaires [5,14] or diagnostic tools [15-17]. Surgery and rehabilitation methods solve most of the somatic issues, but those linked to behavior seem to persist in many people with CS until adulthood [6]. Some investigators have studied autistic-like behaviors in CS, but their

${ }^{\star}$ Correspondence to: Laurence Vaivre-Douret, Hospital Universitaire NeckerEnfants Malades, INSERM UMR 1178/1018 CESP, Carré Necker, Porte N4, 149 rue de Sèvres, 75015 Paris, France, E-mail: laurence.vaivre-douret@inserm.fr

Key words: CHARGE syndrome, autistic traits, sensory impairments, deaf blindness, Challenging behaviors; genetic disease

Received: June 24, 2018; Accepted: June 30, 2018; Published: July 01, 2018 
research only partly answered questions such as the frequency of ASD in CS, whether there is a specific autistic-like profile in CS and what factors lead to the development of ASDs in CS.

No critical review has investigated autistic-like behaviors in CS. This review aimed to report the current knowledge regarding the state of art of ASDs in CS. We describe the studies investigating ASDs and their frequency in people with CS and their results and analyze the factors linked to autistic-like and challenging behaviors in CS.

\section{Methods}

In April 2016, we searched for English or French articles in the databases SCIENCE DIRECT, MEDLINE, and PsycINFO from their inception by using the keywords "autis", "behavior", "development" associated with "CHARGE syndrome". We found 247 articles (Figure 1). The topic of most articles was autism (pharmacology, genetic) and genetic diseases in which ASDs is reported. Thus, we restrained our research to keywords of the title, subject and abstract and found 16 citations: 4 were about autistic $[14,18,19]$ and challenging [5] behaviors and others were about behavior in general $[8,20]$, parental stress [21], sleep disturbances [22], and executive functioning [23] in CS and one was an educators' primer [24]. Two articles were about genetics in ASDs $[25,26]$ and one was on positive aspects of specific diet in a child with CS with "ASD" without any details regarding the diagnostic method [27].

We then searched for articles written by important authors in the field (Hartshorne, Johansson) and found 2 theses that were published $[5,18]$ and studied the special issue of the American Journal of Genetics whose topic was "behavior in CHARGE syndrome" [28] and a book about CS [29]. We then searched all articles that cited articles treating ASDs in CS $[14-16,18,30]$. We found no critical review that used the keywords "autism", "CHARGE syndrome", and "challenging behavior" between 2005 and 2009 [31]. Finally, we retained only 7 articles that described assessing autism or challenging behaviors in people with CS (Figure 1). These articles presented methodological quality as they used specific tools for investigations of ASD and took into account various factors.

\section{Results and discussion}

Description of autistic-like behaviors in CS and their frequency: Hartshorne and Cypher [5] asked parents of 100 children with CS about the psychiatric diagnoses identified in their child: $6 \%$ of the children had a diagnosis of autistic or autistic-like disorder and another $6 \%$ pervasive developmental disorder (PDD). Wachtel et al. [30] asked 87 parents about the psychiatric diagnosis identified in their children with CS: one third mentioned a psychiatric diagnosis and 16\% PDD, including 9\% autism. These methods are questionable because the authors did not assess the children or check in the medical record which tools were used for diagnosis. Moreover, the research depended on establishing a psychiatric diagnosis with people with CS who already have a lot of issues (Table 1). These rates do not seem reliable.

Hartshorne and Cypher [5] developed their own parental questionnaire and included the most frequent behaviors seen in children with attention deficit hyperactivity disorder, ASD, PDD, obsessive-compulsive disorder, or Tourette's syndrome according to specialists of these conditions. The authors estimated the behaviors on a 5-point Likert-scale. In total, 100 parents of people with CS from various countries completed the questionnaire. The 3 most frequent behaviors were "extreme preference for certain toys, food etc." (69\%), "restricted range of interests and/or pre-occupation with one narrow

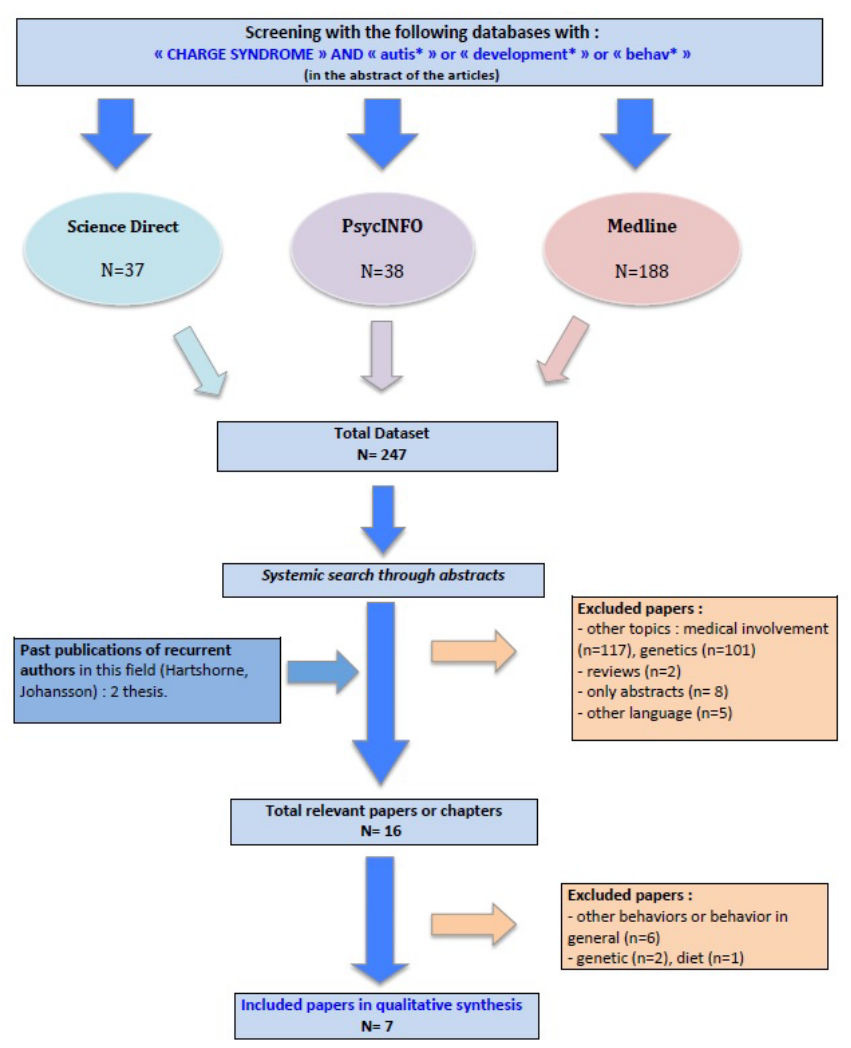

Figure 1. Assessing autism or challenging behaviors in people with CS

interest" (66\%) and "significant difficulty in ability to make same-age friendships" (61\%). This study found a high frequency of autistic-like behaviors in CS but did not compare the participants with a control group of autistic or deafblind people. The questionnaire was not standardized and did not allow for diagnosing autism in CS.

Hartshorne et al. [14] used a standardized and specific parental questionnaire to investigate autistic behaviors, the Autism Behavior Checklist (ABC) [32] and considered $27.5 \%$ of patients with $\mathrm{CS}$ as having autism. The authors reported a specific autistic-like profile. As compared with people with autism, those with CS were more socially engaged [14] and had better language and communication skills [14] because they showed fewer language abnormalities typically manifested by ASD (e.g., echolalia, avoiding pronouns) [14]. As compared with deafblind people, people with CS showed more resistance to change, temper tantrums and ritualistic behaviors [14]. Even if the ABC is standardized and widely used in the field of autism, it has high specificity [33] and can lead to an underestimation of the frequency of ASDs. Moreover, the use of parental questionnaires does not ensure that questions are understood and does not allow the investigator to explain the question or give details. These details can be important for some behaviors and distinguish an appropriate from an inappropriate behavior. Having restrained interests or preoccupation is normal if for instance the person shares these interests with other people. Moreover, with sensory impairments, the diagnosis of ASD and the understanding of behaviors are more complicated. In addition, even if parents know their children well, their subjectivity may impact their judgment.

Johansson et al. [16] used various diagnostic tools for ASDs (i.e., Autism Diagnostic Interview [ADI] [34], DSM-IV) and specific autism scales (i.e., ABC, Childhood Autism Rating Scale [CARS] [35] with 
Table 1. Selected articles for review (ABC: Autism Behavioral Checklist. ADI: Autism Diagnostic Interview. DSM: Diagnostic and Statistical manual of Mental disorders. OAV: OculoAuriculo-Vertebral syndrome. CARS: Childhood Autism Rating Scale. CS: CHARGE syndrome. VABS: Vineland Adaptive Behavior Scale).

\begin{tabular}{|c|c|c|c|c|c|c|}
\hline \multirow{2}{*}{ Reference } & \multirow{2}{*}{ Groups } & \multirow{2}{*}{$\begin{array}{c}\text { Sample } \\
\text { size }\end{array}$} & \multirow{2}{*}{$\begin{array}{c}\text { Age, } \\
\text { years , months } \\
\text { (mean) }\end{array}$} & \multicolumn{3}{|c|}{ Methods of assessment } \\
\hline & & & & Autism diagnose & Challenging behaviors & Characteristics \\
\hline Fernell et al. ${ }^{15}$ & $\mathrm{CS}$ & 3 & - & $\begin{array}{l}\text { DSM-III/IV } \\
\text { ADI } \\
\text { CARS }\end{array}$ & - & Leiter not verbal \\
\hline Hartshorne and Grialou ${ }^{14}$ & $\mathrm{CS}$ & 160 & 10.9 & $\mathrm{ABC}$ & - & - \\
\hline Johansson et al. ${ }^{16}$ & $\mathrm{CS}$ & 25 & 8.11 & $\begin{array}{l}\text { ADI-Revised } \\
\text { DSM-IV }\end{array}$ & - & VABS or Wechsler \\
\hline Johansson et al. ${ }^{18}$ & $\begin{array}{l}\text { CS } \\
\text { Möbius } \\
\text { OAV }\end{array}$ & $\begin{array}{l}25 \\
21 \\
19\end{array}$ & $\begin{array}{l}8.11 \\
8.11 \\
12.4\end{array}$ & $\begin{array}{l}\text { ADI-Revised } \\
\text { DSM-III/IV } \\
\text { CARS } \\
\text { ABC }\end{array}$ & - & - \\
\hline Hartshorne and Cypher ${ }^{5}$ & $\mathrm{CS}$ & 100 & 7 & Reported diagnosis & - & - \\
\hline Wachtel et al. ${ }^{30}$ & $\mathrm{CS}$ & 87 & 11.1 & Reported diagnosis & - & - \\
\hline Vervloed et al. ${ }^{20}$ & $\mathrm{CS}$ & 27 & 12 & - & CBCL & $\begin{array}{c}\text { Temperament scale for mental } \\
\text { retardation } \\
\text { VABS }\end{array}$ \\
\hline
\end{tabular}

25 people with CS. They classified different levels of autism, from childhood autism (with all ADI and DSM criteria) to autistic traits (only 3 to 5 DSM criteria). The study concluded an autism frequency of $68 \%$ among the people studied. This rate may be an overestimation because the authors considered autistic-like behaviors and autistic traits and not just childhood autism. CS is a clinically heterogeneous syndrome, and 25 participants seems a too-small sample to generalize to the whole population of CS. Especially, the authors underlined a recruitment bias in favor of people with more severe hearing and visual impairments. Moreover, direct assessments with, for instance, the Autism Diagnostic Observation Schedule (ADOS) [36] in association with the ADI should be considered to investigate autistic behaviors in CS people as is advised for children [37].

Furthermore, dual sensory impairment induces inappropriate questions when using tools created for people without sensory impairment [38]. The choice of diagnostic tool is important. The ADI seems the most appropriate, but it was used in one study with only a few participants [17]. This lack of studies using diagnostic tools in a large sample of patients is explained by the rarity of CS and its heterogeneity, with sometimes a severe picture that is difficult to assess. Thus, CS people are usually scattered, and assessments with a sufficiently large number of people is difficult.

So, the rate of ASDs in CS has a large range, from $9 \%$ to $68 \%$, $[16,30]$ and is uncertain, depending on the definition and thus needs to be studied in larger populations.

Determinants or risk factors of autism and autistic traits in CS: CS patients can exhibit autistic-like behaviors. Challenging behaviors are not diagnostic criteria of ASDs. Regardless, autistic people are more likely to exhibit challenging behaviors [39-40] probably given their communication difficulties [41] and sensory integration issues [42]. Challenging behaviors are defined as "culturally abnormal behaviors of such an intensity, frequency or duration that the physical safety of the person or others is likely to be placed in serious jeopardy, or behaviors which is likely to seriously limit use of, or result in the person being denied access to, ordinary community facilities" [43]. Distinguishing challenging and autistic-like behaviors in CS is difficult because they can overlap. Few studies have investigated the links between autistic traits and medical or history data.

Vervloed et al. [20] investigated challenging behaviors by using a parental scale for 27 people with CS. They used the scale created by
Hartshorne and Cypher [5] to investigate autistic behaviors and most of the medical involvement scales used by previous authors $[5,44]$. They also accounted for length of hospitalization and number of operations. The authors performed a number of statistical tests among only a few participants, which may have reduced the strength of these tests. People with cerebral abnormalities were more socially withdrawn and seemed to have more difficulties regulating their emotions. However, the authors did not specify the method of brain investigation and the anomalies. Their results did not show any link between those two variables, except that people with heart defects and cerebral abnormalities showed more challenging behaviors. Their results showed less externalized behaviors in people who underwent heart surgery. The authors indicated that the behaviors might be due to fatigue. Other factors must be taken into account to clarify and better explain this result. The length of hospitalization and number of operations did not seem to be significant risk factors for challenging behaviors.

According to the authors of one study, [15] autistic behaviors could be present in CS whatever the degree and association of sensory impairments. Three studies investigated risk factors for autistic-like behaviors $[5,16,28]$, but only one of these used objective tools to assess various deficits [16].

Hartshorne and Cypher [5] investigated factors linked to autistic-like behaviors with their parental questionnaire. The authors accounted for medical involvement by using a severity scale that they developed, attributing one point to each medical condition (e.g., renal abnormalities, heart defects). The greater the medical involvement, the more severe the autistic-like behaviors. The authors also investigated the difference in autistic behaviors in CS in terms of the presence of deafblindness. Being deafblind was defined according to parents' opinion in terms of the severity of the sensory deficits of the child. Some autistic-like behaviors were more frequent in CS patients with than without the deafblind condition. This result suggests a possible specific autistic profile in CS depending on the presence of deafblindness. The authors also found more autistic-like behaviors in younger than older children with CS. The risk factors suggested in this study are relevant, but the measurements of sensory impairments need to be more precise. The judgment of sensory impairment severity by parents is subjective and might be linked to degree of intellectual disability. Thus, intellectual disability and sensory impairment severity could be confounded. Moreover, the severity scale of medical involvement is criticized 
because it attributes the same value to very different somatic anomalies. To improve the validity of the study, the same team of experts [14] used a specific parental questionnaire to investigate autistic-like behaviors. They found the same results as previous regarding the association between deafblindness and autism and between somatic severity and autism. The authors used the same severity scale of sensory impairments and medical involvement, and likely some of their participants were the same as in the previous study. The results indicated that autistic-like behaviors were more frequent in children with CS than in those without CS but with the deafblind condition. Thus, even if deafblindness may be a risk factor, it is not sufficient to explain the development of autism.

Johansson et al. [16] were the only authors who used specific and standardized diagnostic tools. To measure the presence and the severity of autism, they used the DSM, ADI, CARS and ABC. To assess learning disability, they used the Wechsler Intelligence Scale for Children or the Vineland Adaptive Behaviour Scale. To assess sensory impairment, they objectively measured hearing and visual deficiency with specific tests used by ophthalmologists and by ear, nose and throat specialists. They classified visual and auditory deficiency into 3 categories according to the degree of visual acuity and hearing loss. To estimate the neurologic anomalies, the authors collected brain MRI images and brain CT scans for participants. Both visual loss and cerebral anomalies were linked to intellectual outcome, with the level highly correlated with the severity of autistic-like behaviors. Cerebral abnormalities were present in most of the participants, more frequently forebrain midline abnormalities (corpus callosum hypoplasia). White and grey matter abnormalities and forebrain midline abnormalities were more frequent in people with CS and autism (5 DSM-IV criteria) than those with CS with only a few autism traits (3-4 DSM-IV criteria) or no autistic traits [16]. The authors could not perform multivariate analysis but suggested that sensory impairment could be a marker of brain damage in CS, as was hypothesized in deaf [45] and blind [46] people. Thus, this study indicated that learning disabilities, visual deficits, and cerebral anomalies were all associated with severity of autism in CS.

Two studies [14,18] showed autistic behaviors more frequent in children with CS than those with only the deafblind condition. In addition to visual and auditory deficiencies, children with CS had vestibular and olfactory impairments and experienced several traumatic events due to their medical conditions, which could explain the difference in results from previous studies.

\section{Conclusions}

This analysis of the literature showed that children and adults with CS can exhibit autistic-like behaviors whose profile is not the same as with classical autism. The CS behavioral autistic-like profile features better communication skills and more interest in social relationships than with classical autism. Compared to deafblind people, CS people show less self-regulation abilities [14], with more ritualistic, stereotyped and self-stimulation behaviors. To better define this profile, the ADI, which seems to be the most appropriate tool for diagnosing ASD, could be used. A direct assessment of autism, such as with the ADOS [36] could be used given the multiple disabilities of children with CS.

The rate of ASDs in CS varies from 9\% to 68\% [16,30]. Patients with CS more frequently exhibit autistic-like behaviors than do deafblind people, which suggests a specific link between ASDs and CS [17]. Cerebral anomalies [16], visual loss [14,17], medical involvement [5,14] and low intellectual level [16] appear to be relevant risk factors for the development of ASDs in CS. However, we do not know the strength of each factor and any possible overlap. The developmental trajectory of autistic-like behaviors must be investigated in light of the evidence of the impact of age in some studies [5,14]. Executive functioning has been found linked to autistic-like behaviors, $[2,3]$ but its impairment in CS lacks consensus [47].

Few studies have precisely investigated autistic behaviors in CS. Emotion regulation abilities, relationships with relatives, social rules knowledge, theory of mind abilities, and of course anxiety should be investigated in future studies.

\section{Acknowledgment}

This work was supported by the University Institute of France grant funds, number L17P99IUF004.

\section{References}

1. Hall BD (1979) Choanal atresia and associated multiple anomalies. J Pediatr 95: 395398. [Crossref]

2. Hittner H, Hirsch N, Kreh G, Rudolph A (1979) Colobomatous microphthalmia, heart disease, hearing loss, and mental retardation--a syndrome. J Pediatr Ophthalmol Strabismus 16: 122-128.

3. Pagon RA, Graham Jr. JM, Zonana J, Yong S-L (1981) Coloboma, congenital heart disease, and choanal atresia with multiple anomalies: CHARGE association. J Pediatr 99: 223-227.

4. Raqbi F, Le Bihan C, Dureau P, Lyonnet S, Abadie V (2003) Early prognostic factors for intellectual outcome in CHARGE syndrome. Dev Med Child Neurol 45: 483-488.

5. Hartshorne TS, Cypher AD (2004) Challenging Behavior in CHARGE Syndrome. Ment Health Asp Dev Disabil. 2004.

6. Stratton K (2011) Young adulthood. In: CHARGE Syndrome. 2011:381.

7. Bernstein V, Denno LS (2005) Repetitive behaviors in CHARGE syndrome: Differential diagnosis and treatment options. Am J Med Genet A 133: 232-239.

8. Lauger K, Cornelius N, Keedy W (2005) Behavioral features of CHARGE syndrome: Parents' perspectives of three children with CHARGE syndrome. Am J Med Genet A 133: 291-299.

9. Souriau J, Gimenes M, Blouin C, Benbrik I, Benbrik E, et al. (2005) CHARGE syndrome: developmental and behavioral data. Am J Med Genet A 133A: 278-281. [Crossref]

10. APA (2013) DSM 5. Diagnostic and Statistical Manual of Mental Disorders. American Psychiatric Association.

11. APA (2000) DSM-IV-TR: Diagnostic and Statistical Manual of Mental Disorders, Text Revision. American Psychiatric Association

12. Davenport SL, Hefner MA, Mitchell JA (1986) The spectrum of clinical features in CHARGE syndrome. Clin Genet 29: 298-310. [Crossref]

13. APA (1980) DSM-III-R: Diagnostic and Statistical Manual of Mental Disorders. American Psychiatric Association.

14. Hartshorne TS, Grialou TL, Parker KR (2005) Autistic-like behavior in CHARGE syndrome. Am J Med Genet A 133A: 257-261. [Crossref]

15. Fernell E, Olsson VA, Karlgren-Leitner C, Norlin B, Hagberg B, et al. (1999) Autistic disorders in children with CHARGE association. Dev Med Child Neurol 41: 270-272. [Crossref]

16. Johansson M, Rastam M, Billstedt E, Danielsson S, Stromland K, et al. (2006) Autism spectrum disorders and underlying brain pathology in CHARGE association. Dev Med Child Neurol 48: 40-50. [Crossref]

17. Johansson M, Gillberg C, Rastam M (2010) Autism spectrum conditions in individuals with Möbius sequence, CHARGE syndrome and oculo-auriculo-vertebral spectrum: diagnostic aspects. Res Dev Disabil 31: 9-24.

18. Smith IM, Nichols SL, Issekutz K, Blake K (2005) Behavioral profiles and symptoms of autism in CHARGE syndrome: preliminary Canadian epidemiological data. $\mathrm{Am} \mathrm{J}$ Med Genet A 133: 248-256.

19. Vervloed MP, Hoevenaars-van den Boom MA, Knoors H, van Ravenswaaij C, Admiraal RJ (2006) CHARGE syndrome: Relations between behavioral characteristics and medical conditions. Am J Med Genet A 140: 851-862. 
20. Wulffaert J, Scholte EM, Dijkxhoorn YM, Bergman JE, van Ravenswaaij-Arts CM, et al. (2009) Parenting Stress in CHARGE Syndrome and the Relationship with Child Characteristics. J Dev Phys Disabil 21: 301-313. [Crossref]

21. Hartshorne TS, Heussler HS, Dailor AN, Williams GL, Papadopoulos D, et al. (2009) Sleep disturbances in CHARGE syndrome: types and relationships with behavior and caregiver well-being. Dev Med Child Neurol 51: 143-150.

22. Hartshorne TS, Nicholas J, Grialou TL, Russ JM (2007) Executive function in CHARGE syndrome. Child Neuropsychol 13: 333-344. [Crossref]

23. Smith KG, Smith IM, Blake K (2010) CHARGE syndrome: an educators' primer. Educ Treat Child 33: 289-314.

24. Capelli LP, Krepischi AC, Gurgel-Giannetti J, Mendes MF, Rodrigues T, et al. (2012) Deletion of the RMGA and CHD2 genes in a child with epilepsy and mental deficiency. Eur J Med Genet 55: 132-134. [Crossref].

25. Moss, Richards C, Nelson L, Oliver C (2013) Prevalence of autism spectrum disorder symptomatology and related behavioural characteristics in individuals with Down syndrome (English). Autism Lond 17: 390-404. https://frodon.univparis5.fr/url?http:// search.ebscohost.com/login.aspx?direct $=$ true $\& \mathrm{db}=\mathrm{fcs} \& \mathrm{AN}=27524436 \&$ lang $=$ fr\&site $=$ eds-live \&scope $=$ site.

26. Hsu CL, Lin CY, Chen CL, Wang CM, Wong MK (2009) The effects of a gluten and casein-free diet in children with autism: a case report. Chang Gung Med J 32: 459-465. [Crossref]

27. Hartshorne TS, Hefner MA, Davenport SL (2005) Behavior in CHARGE syndrome: introduction to the special topic. Am J Med Genet A 133A: 228-231. [Crossref]

28. Hartshorne TS, Hefner MA, Davenport SLH, Thelin JW (2011) CHARGE Syndrome. Plural Pub.

29. Wachtel LE, Hartshorne TS, Dailor AN (2007) Psychiatric diagnoses and psychotropic medications in CHARGE syndrome: A pediatric survey. J Dev Phys Disabil 19: 471483.

30. Sheriff LK (2012) Parent and teacher perceptions of challenging behavior in their children and students with CHARGE syndrome. 2012.

31. Krug DA, Arick JR, Almond PJ (1988) Autism behavior checklist. Austin TX -Ed.

32. Tordjman S (2002) Les instruments d'évaluation de l'autisme: intérêts et limites. Psychiatr Enfant 45: 533-558.

33. Lord C, Rutter M, Le Couteur A (1994) Autism Diagnostic Interview-Revised: a revised version of a diagnostic interview for caregivers of individuals with possible pervasive developmental disorders. J Autism Dev Disord 24: 659-685.

34. Schopler E, Reichler RJ, DeVellis RF, Daly K (1980) Toward objective classification of childhood autism: Childhood Autism Rating Scale (CARS). J Autism Dev Disord 10: $91-103$.
35. Lord C1, Risi S, Lambrecht L, Cook EH Jr, Leventhal BL, et al. (2000) The autism diagnostic observation schedule-generic: a standard measure of social and communication deficits associated with the spectrum of autism. J Autism Dev Disord 30: 205-223. [Crossref]

36. Le Couteur A, Haden G, Hammal D, McConachie H (2008) Diagnosing autism spectrum disorders in pre-school children using two standardised assessment instruments: the ADI-R and the ADOS. J Autism Dev Disord 38: 362-372. [Crossref]

37. Le Couteur A, Haden G, Hammal D, McConachie H (2008) Diagnosing autism spectrum disorders in pre-school children using two standardised assessment instruments: the ADI-R and the ADOS. J Autism Dev Disord 38: 362-372. [Crossref]

38. de Vaan G, Vervloed MP, Hoevenaars-van den Boom M, Antonissen A, Knoors H, et al (2016) Critical Review of Screening and Diagnostic Instruments for Autism Spectrum Disorders in People with Sensory Impairments in Addition to Intellectual Disabilities J Ment Health Res Intellect Disabil 9: 36-59.

39. Murphy O, Healy O, Leader G (2009) Risk factors for challenging behaviors among 157 children with autism spectrum disorder in Ireland. Res Autism Spectr Disord 3: 474-482.

40. Matson JL, Wilkins J, Macken J (2008) The relationship of challenging behaviors to severity and symptoms of autism spectrum disorders. $J$ Ment Health Res Intellect Disabil 2: 29-44.

41. Carr EG, Durand VM (1985) Reducing behavior problems through functional communication training. J Appl Behav Anal 18: 111-126.

42. Baker, Lane A, Angley MT, Young RL (2008) The relationship between sensory processing patterns and behavioural responsiveness in autistic disorder: A pilot study. $J$ Autism Dev Disord 38: 867-875.

43. Emerson E (2001) Challenging Behaviour: Analysis and Intervention in People with Severe Intellectual Disabilities. Cambridge University Press, USA.

44. Salem-Hartshorne N1, Jacob S (2005) Adaptive behavior in children with CHARGE syndrome. Am J Med Genet A 133A: 262-267. [Crossref]

45. Jure R, Rapin I, Tuchman RF (1991) Hearing-impaired autistic children. Dev Med Child Neurol 33: 1062-1072. [Crossref]

46. Ek U, Fernell E, Jacobson L, Gillberg C (1998) Relation between blindness due to retinopathy of prematurity and autistic spectrum disorders: a population-based study. Dev Med Child Neurol 40: 297-301.

47. Lasserre E, Vaivre-Douret L, Abadie V (2012) Psychomotor and cognitive impairments of children with CHARGE syndrome: Common and variable features. Child Neuropsychol 19: 449-465.

Copyright: (C2018 Vaivre-Douret L. This is an open-access article distributed under the terms of the Creative Commons Attribution License, which permits unrestricted use, distribution, and reproduction in any medium, provided the original author and source are credited. 